\title{
One-way reciprocal spoof surface plasmons and relevant reversible diodelike beaming
}

\author{
Mehmet Mutlu, ${ }^{1,2, *}$ Semih Cakmakyapan, ${ }^{2,3}$ Andriy E. Serebryannikov, ${ }^{4}$ and Ekmel Ozbay ${ }^{1,2,3}$ \\ ${ }^{1}$ Department of Electrical and Electronics Engineering, Bilkent University, 06800 Ankara, Turkey \\ ${ }^{2}$ Nanotechnology Research Center, Bilkent University, 06800 Ankara, Turkey \\ ${ }^{3}$ Department of Physics, Bilkent University, 06800 Ankara, Turkey \\ ${ }^{4}$ Department of Electrical Engineering, E-3, Hamburg University of Technology, D-21071 Hamburg, Germany
}

(Received 21 February 2012; revised manuscript received 25 March 2013; published 15 May 2013)

\begin{abstract}
One-way excitation of spoof surface plasmons (SPs) and strongly pronounced diodelike extraordinary transmission of linearly polarized waves in the beaming regime can be obtained by combining spoof SPs and cross-polarization conversion resonances. The reciprocal composite structure that is suggested to realize this mechanism consists of a symmetric metallic grating with a subwavelength slit and a metamaterial based ultrathin $90^{\circ}$ polarization rotator and, therefore, shows the broken spatial inversion symmetry. In contrast to the earlier studies of SP inspired transmission through subwavelength slits, asymmetric (one-way) beaming is demonstrated at normal incidence and for both $s$ - and $p$-polarized incident waves. Furthermore, as an implication of Lorentz reciprocity, the studied diodelike mechanism is reversible, which manifests itself in that transmission is significant for one of the two opposite illumination directions at $s$ polarization and for the other direction at $p$ polarization. The obtained numerical and experimental results verify the general idea and enable us to select the optimal operation regimes.
\end{abstract}

DOI: 10.1103/PhysRevB.87.205123

PACS number(s): 41.20.Jb, 42.25.Ja, 78.20.Ek, 73.20.Mf

\section{INTRODUCTION}

Surface plasmon (SP) inspired extraordinary transmission (ET) through subwavelength apertures in corrugated metallic slabs has been a subject of extensive study since its first demonstration in 1998. ${ }^{1}$ While classical SPs represent the collective oscillation of a conductor's electron plasma at a conductor/dielectric interface at optical frequencies, their low frequency analogs, spoof or designer SPs, can be obtained even at microwave frequencies by adding corrugations to a metallic slab, or patterning it with subwavelength holes. ${ }^{2,3}$ On the other hand, a wider variety of SP relevant operation regimes can be realized at optical frequencies using structures with corrugations. ${ }^{4}$ It is known that corrugations placed around a sole subwavelength hole or slit on the incidence side can yield a dramatic enhancement of transmission due to the excitation of SPs by means of enabling the matching of the wave vector of an incident wave to that of a supported surface mode. ${ }^{5-7}$ In turn, beaming, i.e., localization of the transmitted beam(s) within desired angular regions of the exit half-space can be achieved owing to placing properly designed corrugations at the exit interface. ${ }^{6-10}$

Asymmetric or unidirectional SPs that appear while Lorentz reciprocity remains probably belong to the most interesting regimes obtainable in metallic gratings. They can be obtained if the conditions of SP excitation at the two sides (left and right) and/or interfaces (front and back) differ. In Refs. 11-13, excitation of unidirectional, i.e., rightward or leftward propagating SPs, has been demonstrated at a corrugated slit-free interface, that has been possible owing to a proper choice of the corrugation and/or incident beam parameters. In Refs. 14 and 15, it is shown that SPs in the structures containing a subwavelength slit can be excited asymmetrically, i.e., on the left and right sides with respect to the slit, or on one of them. In this case, transmission through such a slit is a necessary counterpart of the resulting unidirectional transmission mechanism.
The metallic gratings with a subwavelength slit and identical corrugations on the left and right sides, but differing corrugations at the front- and back-side interfaces can also enable unidirectional excitation of spoof SPs and, thus, strongly asymmetric transmission. ${ }^{16,17}$ To obtain such an asymmetry, $p$-polarized incident waves are required. On the other hand, for such gratings, the SP assisted ET phenomenon has not been observed for $s$-polarized incident waves. However, the diodelike asymmetric beaming regime, i.e., suppression of transmission over the entire range of the observation angle for one of the two propagation directions, requires rather strong tilting. This looks disadvantageous from the point of view of the integration of such structures to optical and microwave circuits. One-way ET connected with nonreciprocal spoof SPs has been studied in Ref. 18 for a patterned metallic surface sandwiched between two magneto-optical dielectric layers. However, in contrast to Refs. 11-17, magnetization is required in this mechanism.

Being a rather general phenomenon, asymmetric transmission is often considered in the context of partial emulation of a diodelike response without using anisotropic or nonlinear constituents. Instead, only isotropic, linear, and passive materials are required. The diffraction relevant asymmetric transmission can be obtained in nonsymmetric photonic ${ }^{19-21}$ and sonic ${ }^{22}$ crystals, and nonsymmetric gratings that contain ultralow-index material layers. ${ }^{23}$ In turn, the polarization conversion relevant mechanism is associated, first of all, with subwavelength and resonator based chiral metamaterials. By using such artificial materials, it has been shown that asymmetry in transmission can be obtained for circularly ${ }^{24-26}$ and linearly ${ }^{27,28}$ polarized incident waves. In particular, an ultrathin structure for the achievement of diodelike asymmetric transmission for incident and outgoing plane waves at the price of extremely strong cross-polarization conversion has recently been suggested. ${ }^{28}$ However, due to its periodic nature in the transverse plane, the field intensity of a transmitted 
wave through this structure is not dependent on the observation angle in the far field. To the best of our knowledge, the studies in Refs. 18-28 have not been connected with the beaming regime.

In this paper, we suggest and experimentally demonstrate a new reciprocal mechanism that enables the one-way excitation of spoof SPs, and relevant unidirectional ET and beaming for normally incident electromagnetic waves. In contrast to the earlier studies dedicated to asymmetric beaming, tilting is not required, while the incident waves may be either $s$ or $p$ polarized. The reversibility of the asymmetric beaming manifests itself in that strong transmission occurs in one of the two opposite directions for $s$ polarization and in the other direction for $p$ polarization.

To realize the proposed mechanism, two counterparts are needed: a symmetric metallic grating with a subwavelength slit for obtaining ET and beaming, and a metamaterial based $90^{\circ}$ polarization rotator for the enabling of the proper breaking of spatial symmetry. Correspondingly, the metallic grating was designed so that the frequency of the strongest ET nearly coincides with the operation frequency of the polarization rotator, at which the strongest cross-polarization conversion and high transmission efficiency co-exist. Compared to the mechanism of obtaining one-way SP excitation proposed in Ref. 18, the composite structure in our case is nonsymmetric, relies on cross-polarization conversion, and does not require magnetization. It is found that the most promising regimes correspond to the thicknesses of the composite structure that are smaller than one free-space wavelength. In the subsequent sections, we will provide the theoretical background along with some design guidelines, present and discuss the simulation and experimental results, and complete the paper with the conclusions.

\section{THEORETICAL BACKGROUND AND DESIGN}

\section{A. General idea}

We envision that the one-way transmission feature, which is relevant to the transmission characteristics of the polarization rotator, allows one obtaining the one-way excitation of spoof SPs and thus, relevant unidirectional ET and beaming, i.e., those existing only for one of the two opposite normal incidence directions for the same polarization state ( $s$ or $p$ ). Accordingly, we target transmitting as much power as possible for the illumination from one side, whereas the transmitted power should be approximately zero for the illumination from the opposite direction, provided that the polarization state is the same. For the sake of definiteness, we refer to the transmission at the incidence from the rotator side as the forward transmission. The backward transmission corresponds to the incidence from the opposite (grating) side.

The general idea of this diodelike transmission mechanism is schematically illustrated in Fig. 1. The resulting structure does not show spatial symmetry in the $z$ direction. An $s$-polarized wave incident on the polarization rotator can be converted to $p$ polarization and transmitted through the subwavelength slit with the assistance of spoof SPs [see Fig. 1(a)]. In turn, an $s$-polarized wave that is directly impinged on the grating side is reflected since spoof SP modes are not supported for this polarization state.

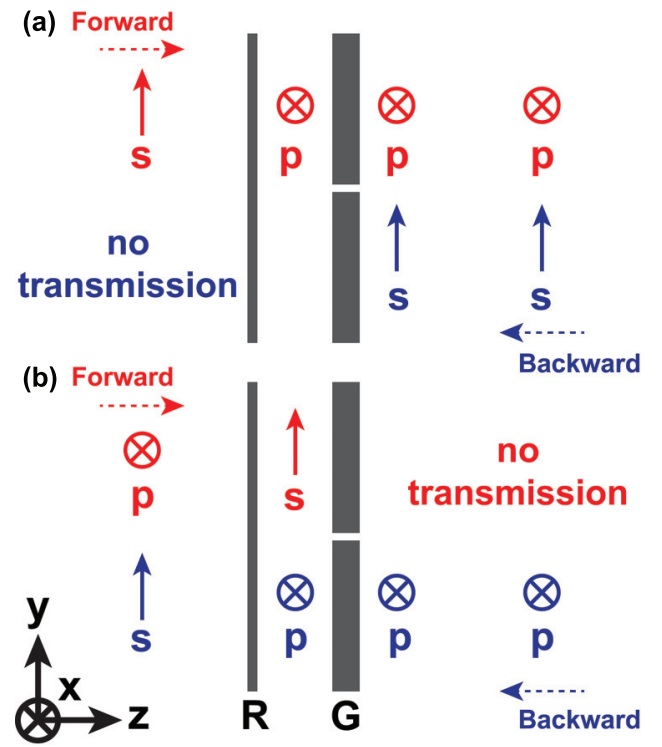

FIG. 1. (Color online) Demonstration of the projected operation principle of the proposed composite structure for (a) $s$ - and (b) $p$ polarized incident waves. The polarization rotator and the metallic grating are denoted by $\mathrm{R}$ and $\mathrm{G}$, respectively. The solid arrows and the crosses show the orientations of the electric field vector for $s$ and $p$-polarized waves, respectively. A dashed arrow indicates the propagation direction of a wave with the same color, i.e., forward or backward.

A $p$-polarized wave incident on the polarization rotator is converted to $s$ polarization and, therefore, cannot be transmitted through the subwavelength slit [see Fig. 1(b)]. On the contrary, a $p$-polarized wave impinged on the grating from the opposite side is transmitted and, afterwards, should be converted to the $s$ polarization with the assistance of the polarization rotator. Furthermore, Fig. 1 shows that the direction of strong transmission can be reversed by simply changing the polarization of the incident light from $s$ to $p$ and vice versa.

In fact, the underlying logic is similar to a big extent to a Faraday-rotator based isolator, a nonreciprocal and widely used device. ${ }^{29}$ However, in contrast to it, the reciprocal structure presented in this paper enables diodelike asymmetric transmission in the beaming regime for $s$ - and $p$-polarized incident waves and, furthermore, at a relatively small thickness of the composite structure, while no magnetization is required. On the other hand, it should be noted that a Faraday isolator can operate when the incident light is unpolarized, while the composite structure presented in this paper operates for particular polarization states of the incident light.

Next, let us briefly discuss the conditions required for obtaining the desired directional selectivity for linearly polarized incident waves. First of all, we take

$$
f_{\mathrm{SP}}=f_{\mathrm{PR}}
$$

where $f_{\mathrm{SP}}$ and $f_{\mathrm{PR}}$ denote the spoof SP and the crosspolarization conversion resonance frequencies, respectively. Since the distance between the grating and the rotator $d$ can be chosen in the proposed designs so that $d \ll \lambda_{\mathrm{PR}}$ (see Sec. III), coupling is expected to occur due to the interaction of the near fields of the structures and the resonance frequency of the composite structure, $f_{\mathrm{CS}} \neq f_{\mathrm{SP}}=f_{\mathrm{PR}}$, in the general case. 
However, the use of Eq. (1) is quite well justified because SP resonances are wide and that gives some freedom in the choice of $f_{\mathrm{SP}}$ and, therefore, in the metallic grating design. Hence, a proper choice of $f_{\mathrm{PR}}$ is the most important.

Despite exhibiting optical activity, the metamaterial based polarization rotator is a reciprocal structure, assuming that only linear and reciprocal materials are going to be used. Assuming propagation in the $+z$ direction, operation of the polarization rotator can be qualitatively characterized in the plane-wave framework by using the Jones matrix formalism as follows:

$$
\left(\begin{array}{c}
E_{p}^{f} \\
E_{s}^{f}
\end{array}\right)=\left(\begin{array}{ll}
T_{p p} & T_{p s} \\
T_{s p} & T_{s s}
\end{array}\right)\left(\begin{array}{c}
E_{p i}^{f} \\
E_{s i}^{f}
\end{array}\right),
$$

where $E_{p i}^{f}$ and $E_{s i}^{f}$ are the $p$ - and $s$-polarized components of the forward propagating incident electric field that correspond to $x$ and $y$ directions, respectively; $T_{p p}$ and $T_{s s}$ are the co-polarization transmission coefficients, and $T_{p s}$ and $T_{s p}$ are the cross-polarization transmission coefficients. Lorentz reciprocity requires that, for incident waves propagating in the $-z$ direction, the transmitted electric field is expressed as $^{30}$

$$
\left(\begin{array}{c}
E_{p}^{b} \\
E_{s}^{b}
\end{array}\right)=\left(\begin{array}{cc}
T_{p p} & -T_{s p} \\
-T_{p s} & T_{s s}
\end{array}\right)\left(\begin{array}{c}
E_{p i}^{b} \\
E_{s i}^{b}
\end{array}\right) .
$$

For an idealized $90^{\circ}$ polarization rotator, we have $T_{p p}=T_{s s}=$ 0 and $T_{s p}=-T_{p s}=1 .^{31}$

The metallic grating with a subwavelength slit exhibits polarization selectivity and a reciprocal response for normally incident waves. The latter means the reception of the same electric field if the transmitter antenna becomes the receiver and vice versa. Thus, the composite structure should also be reciprocal. Then, one may expect that $\left|t_{p p}^{f}\right|=\left|t_{p p}^{b}\right|,\left|t_{s s}^{f}\right|=\left|t_{s s}^{b}\right|$, $\left|t_{s p}^{f}\right|=\left|t_{p s}^{b}\right|$, and $\left|t_{p s}^{f}\right|=\left|t_{s p}^{b}\right|$, where $t_{i j}$ denotes the received electric field when the transmitter antenna emits $j$-polarized radiation and the receiver antenna receives $i$-polarized radiation, and, here, it is assumed that the antennas are aligned such that their main lobe maxima lie in the $\pm z$ directions. ${ }^{32}$ In Sec. III A, this feature will be demonstrated by using the experimental $\left|t_{i j}\right|$ spectra.

\section{B. Choice of structure components}

We utilize the chiral metamaterial based ultrathin $90^{\circ}$ polarization rotator from Ref. 31, which perfectly fulfils the above mentioned requirements. It exhibits the unity transmission of the circularly polarized eigenwaves with a phase difference of $\pi$, so that, contrary to Ref. 28 , there is no sensitivity on the angle of rotation of the polarization plane as long as the wave vector $\mathbf{k}$ of the incident wave is orthogonal to the material plane. The polarization rotator consists of three layers. A unit cell of each of the two resonant layers is composed of four mutually rotated U-shaped split ring resonators. The third layer, a subwavelength mesh that exhibits a negative effective permittivity, is placed between the resonant layers and allows for the obtaining of unity transmission due to the electromagnetic tunneling effect. ${ }^{33,34}$ The two-dimensional metallic grating is assumed to have the same corrugations at front- and back-side interfaces and a single subwavelength slit at the center. To design a proper metallic grating, one of the
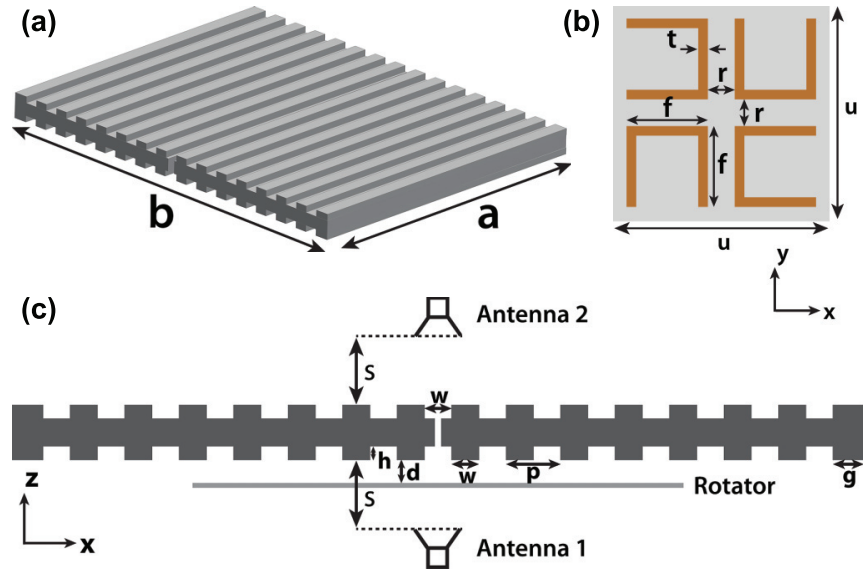

FIG. 2. (Color online) (a) Isometric view of the metallic grating with a subwavelength slit, (b) front layer of the polarization rotator, and (c) schematic of the experimental setup. In plot (c), the distance between the antennas and the grating $S$ is not drawn to scale.

early suggested performances can simply be rescaled for the purpose of satisfying Eq. (1).

Figure 2 shows the grating geometry, a unit cell of one of the two resonant layers of the polarization rotator and the experiment setup. The utilized geometrical parameters for the grating are the following: $a=400 \mathrm{~mm}, b=500 \mathrm{~mm}, w=$ $16 \mathrm{~mm}, p=32 \mathrm{~mm}, h=8 \mathrm{~mm}$, and $g=18 \mathrm{~mm}$. The width and the length of the slit are given as $4 \mathrm{~mm}$ and $16 \mathrm{~mm}$, respectively. For the front layer of the polarization rotator, which is depicted in Fig. 2(b), we take $t=0.7 \mathrm{~mm}, f=6 \mathrm{~mm}$, $r=2 \mathrm{~mm}$, and $u=16 \mathrm{~mm}$. The back layer is obtained by rotating the individual split-ring resonators of the front layer by $90^{\circ}$ and as a result, a chiral structure with fourfold rotational symmetry is obtained. A subwavelength mesh that exhibits negative effective permittivity throughout a wide frequency range is sandwiched between the front and the back layers. Its unit cell is depicted at the inset of Fig. 3. The geometrical parameters for the mesh are given by $c=0.5 \mathrm{~mm}$ and $e=$ $3.2 \mathrm{~mm}$. Figure 2(c) shows the schematic of the experimental setup together with the geometrical parameters of the metallic grating with the subwavelength slit. The distance between the

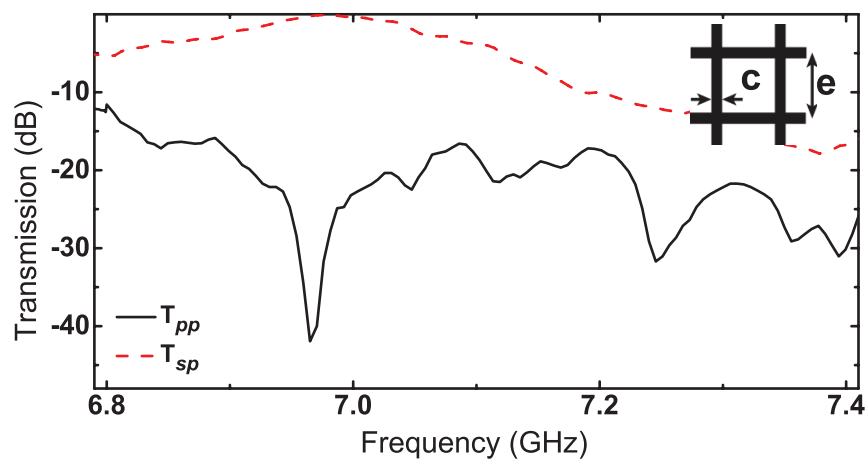

FIG. 3. (Color online) Magnitudes of the experimental linear transmission coefficients for the polarization rotator. The maximum of $\left|T_{s p}\right|$ is observed at $6.98 \mathrm{GHz}$. The inset shows the geometry of the subwavelength mesh sandwiched between the front and back layers of the rotator. 


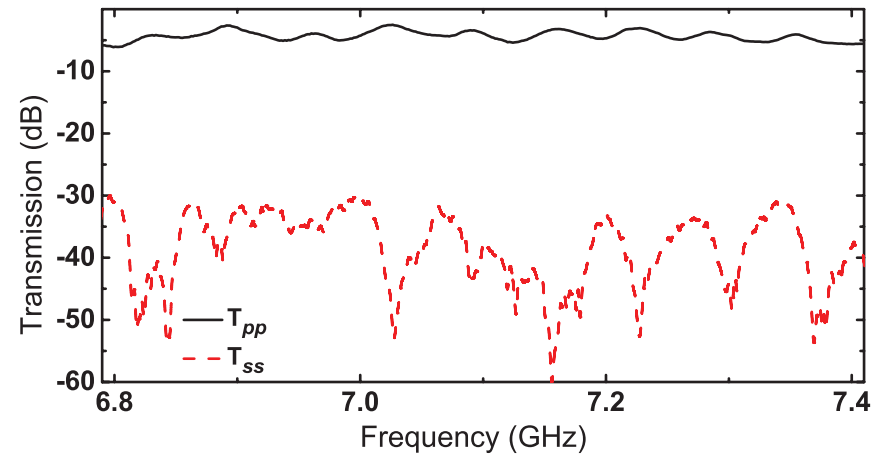

FIG. 4. (Color online) Magnitudes of the experimental linear co-polarized transmission coefficients of the metallic grating with a single subwavelength slit.

metallic grating and the horn antennas is set to $S=400 \mathrm{~mm}$ in the experiments.

The structures were positioned in a symmetric configuration between the antennas. The transmission coefficient measurements were performed by using an Anritsu 37369A network analyzer. For the purposes of the experimental study, we used the polarization rotator with the dimension of 18 by 18 unit cells. In Fig. 3, we provide the magnitudes of the experimental linear transmission coefficients for the polarization rotator, namely $\left|T_{p p}\right|$ (co-polarized component) and $\left|T_{s p}\right|$ (crosspolarized component). For the two other linear transmission coefficients, we have $T_{s s}=T_{p p}$ and $T_{p s}=-T_{s p}$ as mentioned in Sec. II A. According to Fig. 3, $f_{\mathrm{PR}} \simeq 6.98 \mathrm{GHz}$. The structure exhibits a cross-polarization conversion efficiency of $98 \%$ at this frequency.

In the next step, the metallic grating that contains a single subwavelength slit has been characterized experimentally. Figure 4 presents the linear co-polarized transmission coefficients, $T_{p p}$ and $T_{s s}$. The cross-polarization transmission coefficients, $T_{s p}$ and $T_{p s}$, are zero due to the lack of optical activity and, thus, are not shown. At the chosen operation frequency, $f=f_{\mathrm{PR}}=6.98 \mathrm{GHz},\left|T_{p p}\right| \simeq-3.5 \mathrm{~dB}$. On the other hand, $\left|T_{s s}\right|<-30 \mathrm{~dB}$ throughout the frequency range of the measurement, so that the extinction ratio is better than $25 \mathrm{~dB}$ in the vicinity of $7 \mathrm{GHz}$. The simulation results (see $\mathrm{Sec}$. II C) indicate that the electric field is strongly enhanced at the center of the subwavelength slit and that results in a strong coupling between spoof SPs at the front and the back sides. This results in the appearance of ET and beaming, which are known to be absent in case of a corrugation free slab.

\section{Beaming with and without rotator}

Extensive numerical simulations (Computer Simulation Technology Microwave Studio) for $d=5 \mathrm{~mm}$ and normally incident waves in the vicinity of $f=7 \mathrm{GHz}$ have been carried out in order to study the beaming behavior of the transmitted co-polarized field component for the grating and cross-polarized field components for the composite structure.

Assuming that the origin of the $x z$ plane is located at the center of the subwavelength slit, the radial distance of the points of investigation was set to $400 \mathrm{~mm}$. The examples of angular distributions of electric field intensities with respect to the observation angle are shown in Fig. 5. For the grating alone, the well-pronounced ET in the beaming regime is observed for a $p$-polarized normally incident wave [see Fig. 5(a)]. In line with the obtained results, the full width at half maximum (FWHM) is obtained as approximately $12^{\circ}$. The intensity distribution is symmetric with respect to $0^{\circ}$ as a consequence of the symmetry with respect to the slit. In turn, transmission is expected to vanish for $s$-polarized incident waves due to the lack of a surface mode for this polarization state (compare to Fig. 4). Implied by the symmetry with respect to the $x y$ plane at $z=0$, i.e., the center of the slit, transmission properties do not depend on whether the forward or the backward transmission case is considered.

Afterwards, the composite structure, which contains the rotator and the metallic grating, has been investigated for normally incident waves. The beaming regime is achievable now when the incident $s$-polarized wave propagates in the forward direction, i.e., it is impinged directly on the polarization rotator. This results in the appearance of an outgoing wave with a dominant $p$-polarized component, $\left|t_{p s}^{f}\right|$. Figure $5(\mathrm{~b})$ presents the angular intensity distribution of the transmitted $p$-polarized component for an $s$-polarized incidence in the forward direction. It is noteworthy that in spite of the fact that the incident wave traverses the rotator first, the wave is transmitted to the far field through the subwavelength slit. Thus, the resultant spatial features are mainly determined by the spoof SPs at the

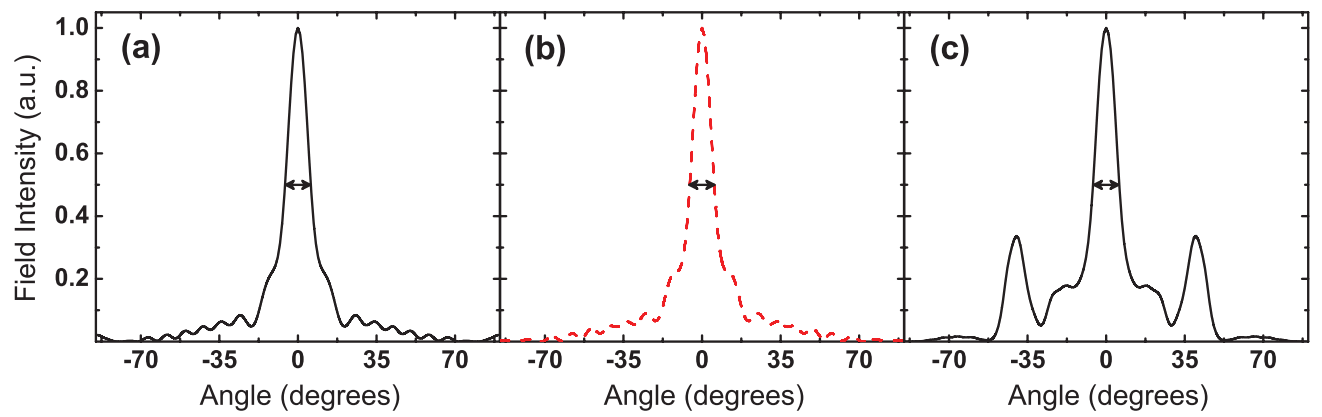

FIG. 5. (Color online) Angular distribution of electric field intensity for $d=5 \mathrm{~mm}$ at a 400 -mm radial distance in the vicinity of $f=7 \mathrm{GHz}$ for (a) the metallic grating with the subwavelength slit alone, and the composite structure at (b) forward and (c) backward illumination. Black solid and red dashed lines indicate that the incident wave is $p$ polarized and $s$ polarized, respectively. The presented results correspond to the $p$-, $p$-, and s-polarized components of the outgoing waves in (a), (b), and (c), respectively. Full widths at half maxima are denoted by the horizontal arrows in (a), (b), and (c). 
exit interface that is in coincidence with the theory of gratings with subwavelength slits. As expected, it is very similar to what is shown in Fig. 5(a). The symmetry with respect to $0^{\circ}$ is observed also in this case, while FWHM $\simeq 12^{\circ}$. For $s$ polarized backward propagating waves, numerical results (not shown here) suggest that the transmission vanishes since spoof SPs cannot be excited at the input side of the metallic grating.

Now let us consider the case when the composite structure is illuminated by a $p$-polarized wave that propagates in the backward direction, being impinged normally on the metallic grating. In this case, the beaming regime is preserved even though the transmission to the far field is performed by the polarization rotator, yielding an outgoing wave with a dominant $s$-polarized component $\left|t_{s p}^{b}\right|$. Figure 5(c) shows the angular distribution of the intensity of the transmitted $s$-polarized component. It is noteworthy that the propagating waves between the polarization rotator and the metallic grating are generally not plane waves with flat phase fronts. However, also in this case, the chiral structure provides the desired rotation while the beaming effect is preserved. In this case, similar to the previous angular distributions, we obtain FWHM $\simeq 12^{\circ}$. It should be noted that, in the backward propagation case, simulation results (not shown here) suggest a nonsymmetric angular distribution with respect to $0^{\circ}$ for the nonsymmetric placement of the rotator with respect to the $y z$ plane at $x=0$. In the backward illumination case, the symmetric placement of the rotator is a significant factor for the obtaining of a symmetric angular distribution, since the outgoing waves are transmitted to the far field by the rotator. Furthermore, we observe from the simulation results (not shown here) that transmission vanishes for a $p$-polarized incident wave propagating in the forward direction.

In addition to the angular intensity distributions, we provide the spatial intensity distributions inside the simulation domain, for the same cases shown in Fig. 5, in Fig. 6 for the purpose of stressing the observed beaming effect. Namely, Figs. 6(a)-6(c) show the intensity distribution of $p$-polarized outgoing waves for $p$-polarized incidence for only grating, $p$-polarized outgoing waves for $s$-polarized incidence for the forward propagation, and $s$-polarized outgoing waves for $p$-polarized incidence for the backward propagation cases, respectively. In Figs. 5 and 6, the intensity distributions for the following cases are not shown since the transmission is found to be negligibly small: (i) $s$-polarized incidence for only grating, (ii) $p$-polarized incidence for the forward propagation, and (iii) $s$-polarized incidence for the backward propagation. The existence of low transmission for these cases is going to be verified by using the experimental results in the next section.

According to Figs. 5 and 6, if a cross-polarized wave is transmitted at $s$-polarized incidence for forward propagation and $p$-polarized incidence for backward propagation, the transmission occurs in the beaming regime, i.e., the transmitted beam is localized within desired angular regions of the exit half-space. It is expected that the diodelike operation in the beaming regime will be obtained by combining this observation with the satisfaction of the following conditions at the operating frequency:

$$
\begin{array}{r}
\left|t_{i j}^{f}\right| \ll\left|t_{p s}^{f}\right|, \quad \text { where }(i, j) \neq(p, s) . \\
\left|t_{i j}^{b}\right| \ll\left|t_{s p}^{b}\right|, \quad \text { where }(i, j) \neq(s, p) .
\end{array}
$$
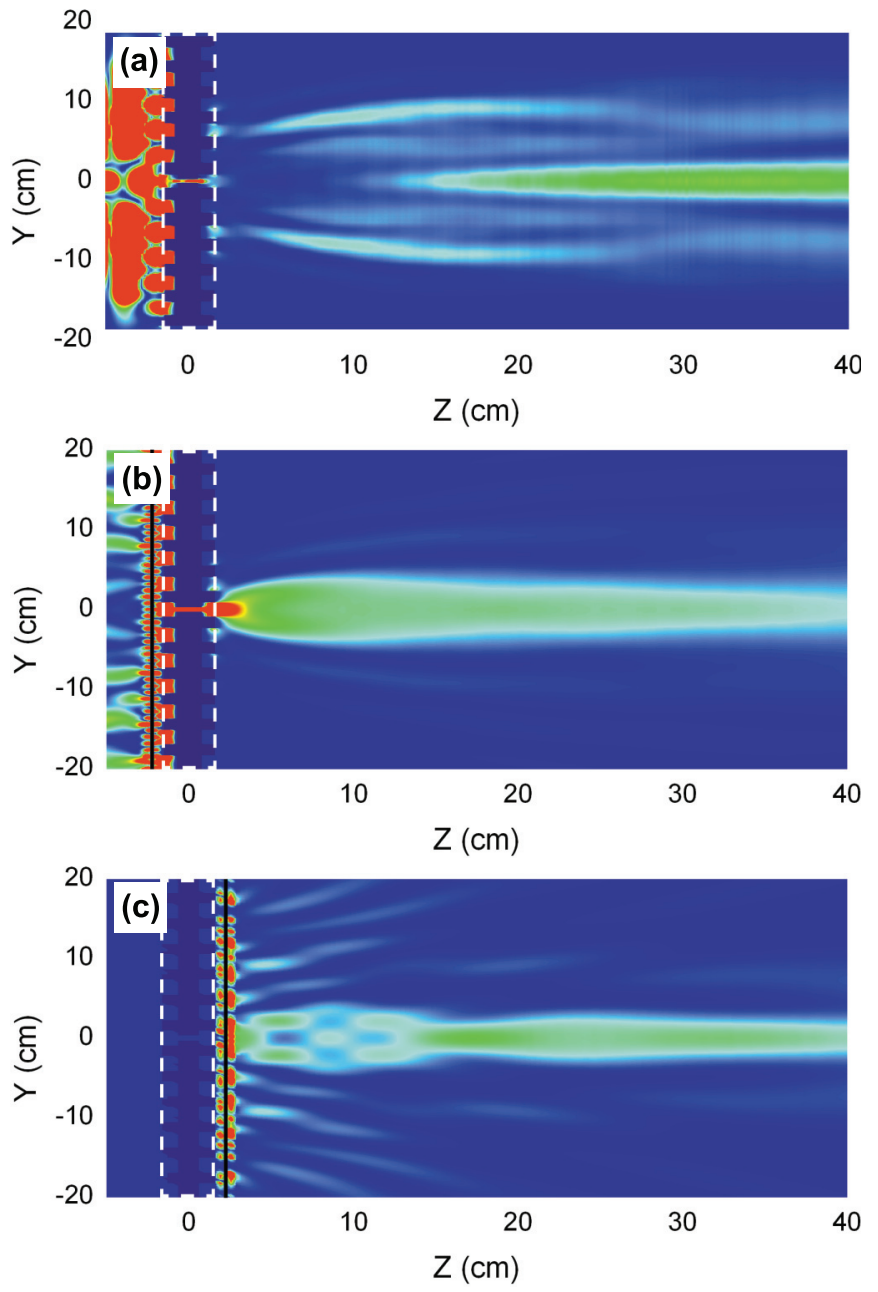

FIG. 6. (Color online) Field intensity distributions of $p$-polarized components for (a) the only grating for $p$-polarized incidence, (b) the composite structure for $s$-polarized forward propagating waves, and (c) field intensity distribution of the $s$-polarized component for $p$-polarized backward propagating waves. The grating with a subwavelength slit is enclosed in a dashed white rectangle and the position of the rotator is shown by a black line.

In addition, Figs. 5 and 6 show that beaming can appear for both forward and backward illumination in the presence of the polarization rotator, but at different polarization states of the incident wave. Indeed, according to Fig. 1, the studied mechanism shows the reversibility of the direction of the diodelike asymmetric transmission, which is implied by Lorentz reciprocity, can be an advantage for practical applications, and may not be present in nonreciprocal systems. Importantly, in this paper, it is demonstrated in the beaming regime. It is seen that the transmission direction of the composite structure can be reversed by a simple change of the incident polarization from $s$ to $p$ polarization and vice versa.

\section{EXPERIMENTAL RESULTS AND DISCUSSION}

\section{A. Experimental transmission results}

An extended experimental study has been performed in order to validate the expected asymmetry in transmission 


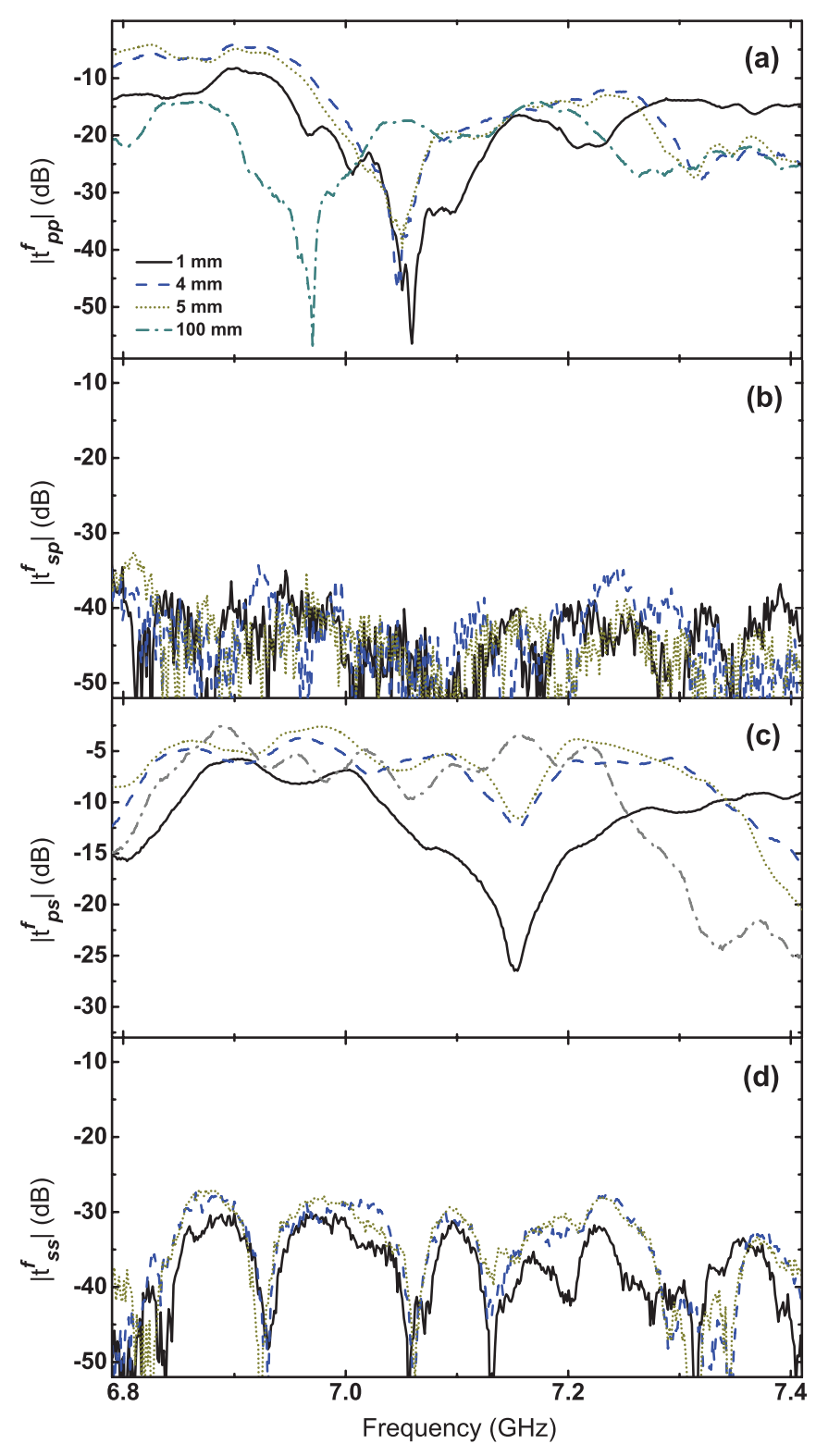

FIG. 7. (Color online) Experimental (a),(d) co-polarized and (b), (c) cross-polarized $\left|t_{i j}^{f}\right|$ spectra. The investigated $d$ values are given in the legend of plot (a).

at normal incidence [see the experiment layout shown in Fig. 2(c)]. Consideration is restricted here to the zero observation angle, which corresponds to the main plane wave component of the angular spectrum of the outgoing beam. As it will be shown subsequently, variation of $d$ allows for controlling the spectra of $\left|t_{i j}\right|$.

Figure 7 presents the forward transmission spectra, $\left|t_{i j}^{f}\right|$. The measurements have been conducted for four values of $d$ as shown in the legend of Fig. 7(a). Two typical cases can be distinguished. For large $d$, i.e., $d=100 \mathrm{~mm}$, the dip of $\left|t_{p p}^{f}\right|$ is located at $f=f_{\mathrm{PR}}=6.98 \mathrm{GHz}$ [see Fig. 7(a)]. For small $d, d \ll \lambda_{\mathrm{PR}}$, the local minimum of $\left|t_{p p}^{f}\right|$ is shifted to approximately $7.05 \mathrm{GHz}$. In the latter case, the interaction of the evanescent waves created by the resonant rotator and the exponentially decaying (in the $z$ direction) spoof SP field at the metal/air interface takes place, leading to the upshift of the resonance frequency. In fact, this indicates the creation of a coupled resonance mode when the distance between these two structures is smaller than the decay lengths of the evanescent fields. It is seen that the shift of the dip in $\left|t_{p p}^{f}\right|$ is significant even when $d$ is decreased from $5 \mathrm{~mm}$ to $1 \mathrm{~mm}$. In the former case, the grating and the rotator are not near-field coupled since $d \simeq 2.3 \lambda_{\mathrm{PR}}$ and thus, the resonance is found at $f_{\mathrm{PR}}$. As expected, both $\left|t_{s p}^{f}\right|$ and $\left|t_{s s}^{f}\right|$ are very small throughout the frequency range of the measurements [see Figs. 7(b) and 7(d)].

According to Fig. 7(c), the cross-polarization conversion (from $s$ to $p$ polarization) is strong in a wide frequency range, while ET of the $p$-polarized wave, which is created by the polarization rotator, is well pronounced. The spectrum of $\left|t_{p s}^{f}\right|$ exhibits maxima in the range that includes the vicinity of $6.98 \mathrm{GHz}$. In reducing the distance between the grating and the rotator, the near-field coupling effects become more pronounced and a transmission dip appears at $f \simeq$ 7.15 GHz. As seen in Fig. 7(c), the dip originating due to the near-field coupling is the most significant when $d=$ $1 \mathrm{~mm}$. In this case, the cross-polarization conversion almost disappears.

The experimental backward transmission spectra, $\left|t_{i j}^{b}\right|$, are shown in Fig. 8. According to the presented results, the reciprocity assumption for the composite structure is validated, which states that $\left|t_{p p}^{f}\right|=\left|t_{p p}^{b}\right|,\left|t_{s s}^{f}\right|=\left|t_{s s}^{b}\right|,\left|t_{s p}^{f}\right|=\left|t_{p s}^{b}\right|$, and $\left|t_{p s}^{f}\right|=\left|t_{s p}^{b}\right|$ (see the discussion in Sec. II A). Among the possible reasons for the very small differences between the corresponding $\left|t_{i j}^{f}\right|$ and $\left|t_{i j}^{b}\right|$ spectra, one should mention the diffraction effects from the sharp edges, multireflections inside the experiment region, and human related alignment errors, such as the possible small displacement of an antenna between the $\left|t_{i j}^{f}\right|$ and $\left|t_{i j}^{b}\right|$ measurements.

The obtained results together show that the conditions given in Eq. (4), which are required for the obtaining of the diodelike beaming feature, are satisfied in the regimes where $\left|t_{p p}^{f}\right| \ll\left|t_{p s}^{f}\right|$ (Fig. 7) and $\left|t_{p p}^{b}\right| \ll\left|t_{s p}^{b}\right|$ (Fig. 8), as occurs in the vicinity of $f=6.98 \mathrm{GHz}$ for $d=100 \mathrm{~mm}$ and in the vicinity of $f \simeq 7.05 \mathrm{GHz}$ for $d \ll \lambda_{\mathrm{PR}}$. Hence, in the above mentioned regimes, the reversibility feature of the composite structure is apparent such that the transmission only occurs at $p$ polarization for $s$-polarized incident waves in the forward direction and at $s$ polarization for $p$-polarized incident waves in the backward direction.

\section{B. Choice of operation frequency}

As follows from the transmission results shown in Figs. 7 and 8 , the obtaining of maximal cross-polarized components $\left(\left|t_{p s}^{f}\right|\right.$ and $\left.\left|t_{s p}^{b}\right|\right)$, on the one hand, and minimal co-polarized components $\left(\left|t_{p p}^{f}\right|\right.$ and $\left.\left|t_{p p}^{b}\right|\right)$, which is necessary to obtain the desired cross-polarization conversion in transmission, on the other hand, do not necessarily occur at the same frequency as a consequence of near-field coupling for $d \ll \lambda_{\mathrm{PR}}$. In other words, the maximum axial ratio and the maximum crosspolarized transmission do not occur at the same frequency for such $d$ values. Thus, the operation frequency should be properly selected on the compromise basis. For the sake of definiteness, we use the data in Fig. 8, which correspond to 


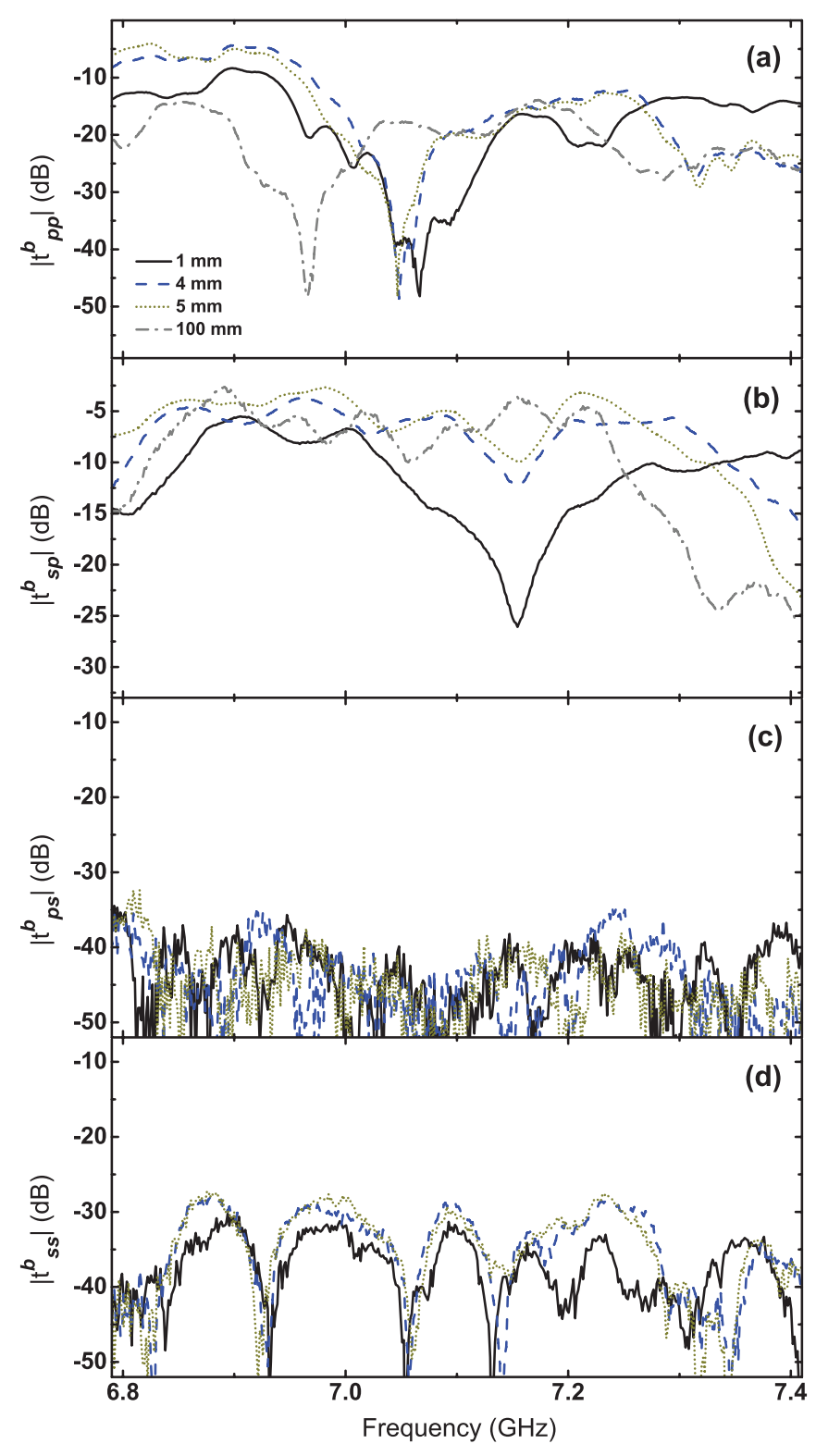

FIG. 8. (Color online) Experimental (a),(d) co-polarized and (b), (c) cross-polarized $\left|t_{i j}^{b}\right|$ spectra. The investigated $d$ values are given in the legend of plot (a).

the backward transmission case. First, the axial ratio $\left|t_{s p}^{b}\right| /\left|t_{p p}^{b}\right|$ must be evaluated. Using the obtained result of this evaluation, a figure-of-merit (FOM) can be introduced as follows:

$$
\mathrm{FOM}=-\frac{\log _{10}\left(\left|t_{s p}^{b}\right| /\left|t_{p p}^{b}\right|\right)}{\log _{10}\left(\left|t_{s p}^{b}\right|\right)} .
$$

Particularly, a larger FOM is achieved for the same axial ratio, but larger cross-polarized transmission. Similarly, we obtain a larger FOM for the same cross-polarized transmission, but larger axial ratio. Furthermore, the defined FOM parameter can be considered as a measure of the forward-to-backward transmission ratio (FBTR). Specifically, in the cases where $\left|t_{p p}^{b}\right| \ll\left|t_{s p}^{b}\right|$ (or $\left.\left|t_{p p}^{f}\right| \ll\left|t_{p s}^{f}\right|\right)$, one would expect obtaining a large FOM value. Considering the regimes, in which $\left|t_{p p}^{f}\right|$ and

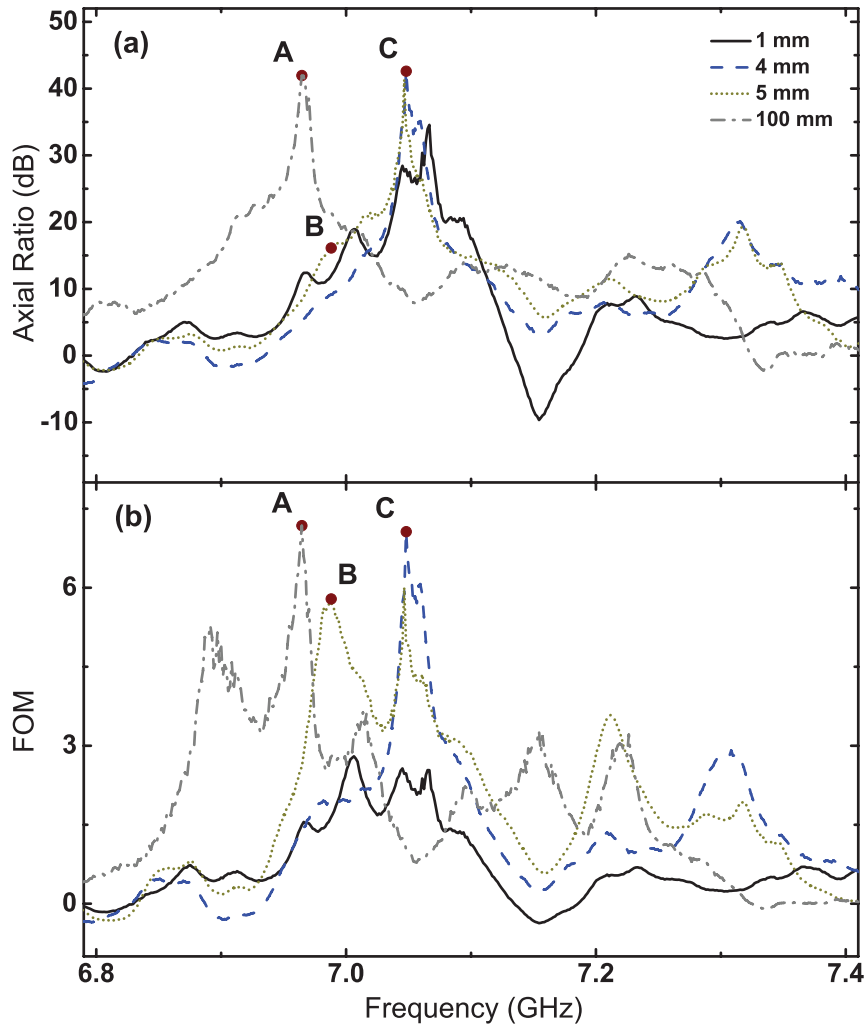

FIG. 9. (Color online) (a) Experimental axial ratio of the transmitted waves and (b) the FOM parameter obtained from Eq. (5).

$\left|t_{p p}^{b}\right|$ are negligibly small, we obtain the following:

$$
\begin{aligned}
\operatorname{FBTR}^{s} & \simeq\left|t_{p s}^{f}\right| /\left|t_{p s}^{b}\right|, \\
\operatorname{FBTR}^{p} & \simeq\left|t_{s p}^{b}\right| /\left|t_{s p}^{f}\right|,
\end{aligned}
$$

where the superscript of FBTR denotes the polarization state of the incident wave. For instance, using Eq. (6), we obtain $\mathrm{FBTR}^{s} \simeq \mathrm{FBTR}^{p} \simeq 40 \mathrm{~dB}$ for $d=5 \mathrm{~mm}$ and $6.95 \mathrm{GHz} \leqslant$ $f \leqslant 7.08 \mathrm{GHz}$.

According to the discussion in the previous paragraph, the FOM parameter may serve as a measure of optimization. If desired, FOM for the forward transmission case can be evaluated by replacing $\left|t_{s p}^{b}\right|$ with $\left|t_{p s}^{f}\right|$ and $\left|t_{p p}^{b}\right|$ with $\left|t_{p p}^{f}\right|$ in Eq. (5). As an implication of Lorentz reciprocity, the two FOM spectra are expected to be equal within the tolerance of experimental errors.

Figure 9 shows the evaluated axial ratio together with the FOM calculated according to the aforementioned definition. The three best pronounced peaks in the FOM spectra are denoted by A, B, and C. They are located at 6.98, 6.99, and $7.05 \mathrm{GHz}$, respectively. The first peak denoted by A corresponds to the vanishing co-polarized transmission that occurs at $f=f_{\mathrm{PR}}$ and for $d=100 \mathrm{~mm}$, i.e., when near-field coupling between the grating and the rotator is not present. The main disadvantage of this case is the large electrical thickness of the composite structure, which is now approximately $3.1 \lambda$. The peak denoted by B $(d=5 \mathrm{~mm})$ corresponds to the maximum cross-polarized transmission among all the $d$ values. On the other hand, the axial ratio is approximately $16 \mathrm{~dB}$ in this case, so that it might provide a reasonable 
compromise between strong cross-polarized transmission and large axial ratio. It is noteworthy that at the peak $\mathrm{B}$ in Fig. $9,\left|t_{s p}^{b}\right|=-2.8 \mathrm{~dB}$. The remaining peak, denoted by $\mathrm{C}$ ( $d=4 \mathrm{~mm}$ ), corresponds to the resonance that is strongly shifted due to the creation of a coupled resonance mode. In this case, an axial ratio of $42 \mathrm{~dB}$ is achieved, i.e., it is rather close to that for the peak A, whereas $\left|t_{s p}^{b}\right| \simeq-6 \mathrm{~dB}$. Accordingly, $\left|t_{s p}^{b, 5 m m}\right| /\left|t_{s p}^{b, 4 m m}\right| \simeq 1.45$. A decision in favor of one of the three operation frequencies should depend on the requirements on concrete performance. For the peaks B and C, the electrical thickness of the composite structure is approximately $0.92 \lambda$ and $0.90 \lambda$, respectively. Thus, the reversible diodelike beaming can be achieved in a compact performance that is thinner than one free-space wavelength.

\section{CONCLUSIONS}

To summarize, we demonstrated a new approach to enable the unidirectional excitation of spoof SPs and the relevant asymmetric ET and beaming. It can be realized in a composite structure that combines a symmetric metallic grating with a single subwavelength slit and a $90^{\circ}$ polarization rotator. This is a good example of how the integration of structures with substantially different properties allows one obtaining new functionalities beyond those associated with each of the individual structures. From the point of view of the metallic grating with a subwavelength slit, the used stacking of the two structures allows the one-way excitation of spoof SPs and the relevant diodelike transmission effect without utilizing anisotropic or nonlinear materials and, furthermore, at normal incidence. For the composite structure, ET can be obtained for s-polarized incident waves that would be impossible while using the grating alone. From the point of view of the metamaterial based $90^{\circ}$ polarization rotator, the diodelike beaming regime would be impossible without stacking with a metallic grating having a subwavelength slit. Reversibility, i.e., ability of the suggested structure to work like a diode in the beaming regime at either $s$ or $p$ polarization, depending on whether the forward or the backward case is considered, is very important. It is noteworthy that it either cannot or is difficult to be realized using most of the other known approaches. Clearly, neither reversibility nor diodelike asymmetric transmission would be possible if the spatial inversion symmetry of the composite structure is not broken properly, e.g., if sandwiching the metallic grating between two identical $90^{\circ}$ polarization rotators. This mechanism is also expected to be realizable in similar composite structures with a total thickness less than $\lambda / 4$. Despite that the performance we used here to demonstrate the suggested mechanism has been designed for microwave frequencies, the main ideas and the results of this paper can be utilized as guidelines for future terahertz and optical frequency designs, which should be a subject of a forthcoming paper.

\section{ACKNOWLEDGMENTS}

This work is supported by the projects DPT-HAMIT, ESFEPIGRAT, EU-N4E, and NATO-SET-181, and TUBITAK under Projects No. 107A004, No. 107A012, and No. 109E301. A.E.S. thanks Deutsche Forschungsgemeinschaft for partial support of this work under Project No. SE1409-2/2. One of the authors (E. Ozbay) also acknowledges partial support from the Turkish Academy of Sciences. *mutlu@ee.bilkent.edu.tr

${ }^{1}$ T. W. Ebessen, H. J. Lezec, T. F. Ghaemi, T. A. Thio, and P. A. Wolf, Nature (London) 391, 667 (1998).

${ }^{2}$ J. Pendry, L. Martin-Moreno, and F. Garcia-Vidal, Science 305, 847 (2004).

${ }^{3}$ A. Hibbins, B. Evans, and J. Sambles, Science 308, 670 (2005).

${ }^{4}$ H. Raether, Surface Plasmons on Smooth and Rough Surfaces and on Gratings (Springer, Berlin, 1988).

${ }^{5}$ T. Thio, K. M. Pellerin, R. A. Linke, H. J. Lezec, and T. W. Ebessen, Opt. Lett. 26, 1972 (2001).

${ }^{6}$ H. J. Lezec, A. Derigon, E. Devaux, R. A. Linke, L. Martin-Moreno, F. J. Garcia-Vidal, and T. W. Ebessen, Science 297, 820 (2002).

${ }^{7}$ H. Caglayan, I. Bulu, and E. Ozbay, Opt. Express 13, 1666 (2005).

${ }^{8}$ A. Derigon and T. W. Ebessen, Opt. Express 12, 3694 (2004).

${ }^{9}$ E. Ozbay, Science 311, 189 (2006).

${ }^{10}$ S. Kim, H. Kim, Y. Lim, and B. Lee, Appl. Phys. Lett. 90, 051113 (2007).

${ }^{11}$ N. Bonod, E. Popov, L. F. Li, and B. Chernov, Opt. Express 15, 11427 (2007)

${ }^{12}$ I. P. Radko, S. I. Bozhevolnyi, G. Brucoli, L. Martin-Moreno, F. J. Garcia-Vidal, and A. Boltasseva, Opt. Express 17, 7228 (2009).

${ }^{13}$ A. Roszkiewicz and W. Nasalski, J. Phys. B 43, 185401 (2010).
${ }^{14}$ S. B. Choi, D. J. Park, Y. K. Leong, Y. C. Yun, M. S. Leong, C. C. Byeon, J. H. Kang, Q. H. Park, and D. S. Kim, Appl. Phys. Lett. 94, 063115 (2009).

${ }^{15}$ F. Lopez-Tejeira, S. G. Rodrigo, L. Martin-Moreno, F. J. GarciaVidal, E. Devaux, T. W. Ebessen, J. R. Krenn, I. P. Radko, S. I. Bozhevolnyi, M. U. Gonzalez, J. C. Weeber, and A. Dereux, Nat. Phys. 3, 324 (2007).

${ }^{16}$ S. Cakmakyapan, A. E. Serebryannikov, H. Caglayan, and E. Ozbay, Opt. Lett. 35, 2597 (2010).

${ }^{17}$ S. Cakmakyapan, H. Caglayan, A. E. Serebryannikov, and E. Ozbay, Appl. Phys. Lett. 98, 051103 (2011).

${ }^{18}$ A. B. Khanikaev, S. H. Mousavi, G. Shvets, and Y. S. Kivshar, Phys. Rev. Lett. 105, 126804 (2010).

${ }^{19}$ A. E. Serebryannikov, Phys. Rev. B 80, 155117 (2009).

${ }^{20}$ A. E. Serebryannikov, A. O. Cakmak, and E. Ozbay, Opt. Express 20, 14980 (2012).

${ }^{21}$ A. O. Cakmak, E. Colak, A. E. Serebryannikov, and E. Ozbay, Opt. Express 18, 22283 (2010).

${ }^{22}$ X.-F. Li, X. Ni, L. Feng, M.-H. Lu, C. He, and Y.-F. Chen, Phys. Rev. Lett. 106, 084301 (2011).

${ }^{23}$ A. E. Serebryannikov and E. Ozbay, Opt. Express 17, 13335 (2009).

${ }^{24}$ R. Singh, E. Plum, C. Menzel, C. Rockstuhl, A. K. Azad, R. A. Cheville, F. Lederer, W. Zhang, and N. I. Zheludev, Phys. Rev. B 80, 153104 (2009). 
${ }^{25}$ V. A. Fedotov, P. L. Mladyonov, S. L. Prosvirnin, A. V. Rogacheva, Y. Chen, and N. I. Zheludev, Phys. Rev. Lett. 97, 167401 (2006).

${ }^{26}$ V. A. Fedotov, A. S. Schwanecke, N. I. Zheludev, V. V. Khardikov, and S. L. Prosvirnin, Nano Lett. 7, 1996 (2007).

${ }^{27}$ C. Menzel, C. Helgert, C. Rockstuhl, E.-B. Kley, A. Tünnermann, T. Pertsch, and F. Lederer, Phys. Rev. Lett. 104, 253902 (2010).

${ }^{28}$ M. Mutlu, A. E. Akosman, A. E. Serebryannikov, and E. Ozbay, Phys. Rev. Lett. 108, 213905 (2012).

${ }^{29}$ B. E. A. Saleh and M. C. Teich, Fundamentals of Photonics (Wiley, New York, 2007).

${ }^{30}$ C. Menzel, C. Rockstuhl, and F. Lederer, Phys. Rev. A 82, 053811 (2010).
${ }^{31}$ M. Mutlu and E. Ozbay, Appl. Phys. Lett. 100, 051909 (2012).

${ }^{32}$ This does not mean that the field has the same angular distribution in the exit half-space, when the structure is illuminated from the side of the rotator and from the side of the metallic grating at the same angle. The possible difference between them belongs to the basic features of the structures with the broken spatial inversion symmetry, which are associated with asymmetric transmission; see Refs. 16 and 19.

${ }^{33}$ L. Zhou, W. Wen, C. T. Chan, and P. Sheng, Phys. Rev. Lett. 94 243905 (2005).

${ }^{34}$ G. Castaldi, I. Gallina, V. Galdi, A. Alù, and N. Engheta, Phys. Rev. B 83, 081105(R) (2011). 